\title{
Critical Current Distributions of Recent Bi-2212 Round Wires
}

\author{
Shaon Barua, Daniel Davis, Yavuz Oz, Jianyi Jiang, Senior Member, IEEE, \\ Eric E. Hellstrom, Senior Member, IEEE, Ulf P. Trociewitz, Senior Member, IEEE, \\ and David C. Larbalestier, Fellow, IEEE
}

\begin{abstract}
Bi}_{2} \mathrm{Sr}_{2} \mathrm{CaCu}_{2} \mathrm{O}_{8+\mathrm{x}}(\mathrm{Bi}-2212)$ is the only high-field, high-temperature superconductor (HTS) capable of reaching a critical current density $J_{c}(16 \mathrm{~T}, 4.2 \mathrm{~K})$ of $6500 \mathrm{~A} \cdot \mathrm{mm}^{-2}$ in the highly desirable round wire (RW) form. However, state-of-theart Bi-2212 conductors still have a critical current density $\left(J_{c}\right)$ to depairing current density $\left(J_{\mathrm{d}}\right)$ ratio around 20 to 30 times lower than that of state-of-the-art $\mathrm{Nb}-\mathrm{Ti}$ or REBCO. Previously, we have shown that recent improvements in Bi-2212 RW $J_{\mathrm{c}}$ are due to improved connectivity associated with optimization of the heat treatment process, and most recently due to a transition to a finer and more uniform powder manufactured by Engi-Mat. One quantitative measure of connectivity may be the critical current $\left(I_{c}\right)$ distribution, since the local $I_{c}$ in a wire can vary along the length due to variable vortex-microstructure interactions and to factors such as filament shape variations, grain-to-grain connectivity variations and blocking secondary phase distributions. Modeling the experimental $V-I$ transition measured on a low resistance shunt as a complex sum of voltage contributions of individual filament and wire sub-sections allows a numerical extraction of the $I_{\mathrm{c}}$ distribution from the $d^{2} V / d I^{2}$ treatment of the $V-I$ curves. Here we compare $\sim 0.1 \mathrm{~m}$ length $I_{\mathrm{c}}$ distributions of $\mathrm{Bi}$-2212 RWs with recent state-of-the-art very high- $J_{c}$ Engi-Mat powder and lower $J_{c}$ and older Nexans granulate powder. We do find that the $I_{\mathrm{c}}$ spread for $\mathrm{Bi}-2212$ wires is about twice the relative standard of high- $J_{c} \mathrm{Nb}-$ Ti well below $H_{\text {irr. }}$. We do not yet see any obvious contribution of the Bi-2212 anisotropy to the $I_{\mathrm{c}}$ distribution and are rather encouraged that these $\mathrm{Bi}-2212$ round wires show relative $I_{\mathrm{c}}$ distributions not too far from high- $J_{\mathrm{c}} \mathrm{Nb}-\mathrm{Ti}$ wires.
\end{abstract}

Index Terms-Bi-2212, Critical current distribution, HTS.

\section{INTRODUCTION}

$\mathbf{R}$ CENT advances in the critical current density $\left(J_{\mathrm{c}}\right)$ of powder-in-tube $\mathrm{Bi}-2212$ round wires have generated great interest for their use in high field magnet technology [1]. An irreversibility field $\left(H_{\text {irr }}\right)$ of more than $100 \mathrm{~T}$ at

Manuscript receipt and acceptance dates will be inserted here. The work is supported by the US DOE Office of High Energy Physics under grant number DE-SC0010421 and by the NHMFL, which is supported by NSF under Award Number DMR-1644779, and by the State of Florida, and is amplified by the U.S. Magnet Development Program (MDP). (Corresponding author: Shaon Barua)

S. Barua is with the Applied Superconductivity Center, National High Magnetic Field Laboratory, Tallahassee, FL 32310, USA and also with Florida State University (e-mail: sbarua@asc.magnet.fsu.edu).

D. S. Davis, Y. Oz, J. Jiang, E. E. Hellstrom, and U. P. Trociewitz are with National High Magnetic Field Laboratory, Florida State University, Tallahassee, FL 32310, USA.

E. E. Hellstrom, and D. C. Larbalestier are with the National High Magnetic Field Laboratory, Florida State University, Tallahassee, FL 32310, USA and also with the Department of Mechanical Engineering, FAMU-FSU College of Engineering.

Digital Object Identifier 10.1109/TASC.2021.3055479
$4.2 \mathrm{~K}$, along with its macroscopically isotropic, twisted, multifilamentary architecture make Bi-2212 a promising conductor for applications above $25 \mathrm{~T}$, where the low temperature superconductors (LTS) $\mathrm{Nb}-\mathrm{Ti}$ and $\mathrm{Nb}_{3} \mathrm{Sn}$ are limited by their much lower irreversibility fields of $\sim 11 \mathrm{~T}$ and $\sim 25 \mathrm{~T}$ (at $4.2 \mathrm{~K})$ [2], [3]. Although its $J_{\mathrm{c}}(16 \mathrm{~T}, 4.2 \mathrm{~K})=6500 \mathrm{~A} \cdot \mathrm{mm}^{-2}$ far exceeds the Future Circular Collider (FCC) specification of $J_{\mathrm{c}}(16 \mathrm{~T}, 4.2 \mathrm{~K})=1500 \mathrm{~A} \cdot \mathrm{mm}^{-2}, J_{\mathrm{c}}$ for state-of-the-art Bi-2212 is still lower than $1 \%$ of the depairing current density [4] $\left(J_{\mathrm{d}} \sim H_{\mathrm{c}} / \lambda \sim 3 \times 10^{6} \mathrm{~A} \cdot \mathrm{mm}^{-2}\right.$, where the thermodynamic critical field $H_{\mathrm{c}} \sim 1 \mathrm{~T}[5]$ and the penetration depth $(\lambda)$ of Bi-2212 $240 \mathrm{~nm}$ ), as opposed to the $20-30 \%$ values achieved with $\mathrm{Nb}-\mathrm{Ti}$ or REBCO. Our recent comprehensive survey of many Bi-2212 wires made between 2009 and 2019 has shown that while $J_{\mathrm{c}}$ defined by $I_{\mathrm{c}} / A$, where $A$ is the total Bi-2212 cross-section, varied by almost a factor of 6 they actually all had almost identical normalized $J_{\mathrm{c}}(H)$, leading us to conclude that very similar vortex pinning properties were shared by all wires and that $\left|J_{\mathrm{c}}\right|$ was determined by the effective filament connectivity [6]. Filament connectivity is affected by multiple factors on many length scales, including filament cross-section variations before reaction and post-reaction defects such as, cracks, voids, blocking secondary phases, and grain-to-grain connectivity variation. Moreover, cuprate grain boundary $J_{\mathrm{c}}$ drops exponentially with increasing grainboundary misorientation angle. Indeed, grain misorientation has historically been the main impediment to the realization of high $J_{\mathrm{c}}$ in cuprate HTSs [1]. Although the quasi-biaxial texture produced by the RW Bi-2212 heat treatment process is believed to mitigate this high angle grain boundary limitation [7], the $c$ - axis rotation of the highly anisotropic Bi-2212 grains results in an inherent $J_{\mathrm{c}}$ variation along the length of each filament. Another commonly observed wire defect is variation of the filament cross section due to the highly non-uniform grain growth characteristics of $\mathrm{Bi}-2212$ from the liquid state. More recently we have seen that the grain texture and filament structure in Bi-2212 RWs are strongly dependent on powder type [4], heat treatment parameters [8], and wire diameter [9].

The $I_{\mathrm{c}}$ distribution is a potential quantitative measure of this complex connectivity variation. We are motivated by the possibility that this distribution can identify avenues for improving $I_{\mathrm{c}}$ and perhaps for wire production quality control. Recently, a transition to finer powder made by Engi-Mat has more than doubled $J_{\mathrm{c}}$ in state-of-the-art Bi-2212 wires [4] compared to earlier wires made with Nexans granulate powder. 
TABLE I

Sample Specifications

\begin{tabular}{lllllllll}
\hline \hline Sample & Material & Powder & Sheath & Manufacturer & Diameter & No. of & Filling \\
Material & & $\begin{array}{l}J_{\mathrm{c}}(5 \mathrm{~T}, 4.2 \mathrm{~K}) \\
{[\mathrm{mm}]}\end{array}$ & Filaments & $\begin{array}{l}\text { Factor } \\
{\left[\mathrm{A} \cdot \mathrm{mm}^{-2}\right]}\end{array}$ \\
\hline $\mathrm{pmm180410}$ & $\mathrm{Bi}-2212$ & Engi-Mat & $\mathrm{Ag}-0.2 \mathrm{wt} . \% \mathrm{Mg}$ & B-OST & 1.0 & $85 \times 18$ & 0.201 & 7115 \\
$\mathrm{pmm100913}$ & $\mathrm{Bi}-2212$ & Nexans & $\mathrm{Ag}-0.2 \mathrm{wt} \% \mathrm{Mg}$ & B-OST & 0.8 & $37 \times 18$ & 0.221 & 4085 \\
$\mathrm{Nb}-\mathrm{Ti}$ & $\mathrm{Nb}-47 \mathrm{wt} \% \mathrm{Ti}$ & & $\mathrm{Cu}$ & Supercon Inc. & 0.6 & 54 & 0.436 & 3280 \\
\hline \hline
\end{tabular}

To extract the $I_{\mathrm{c}}$ distribution of our wires, we employed the method previously employed to characterize the $I_{\mathrm{c}}$ distribution from $V-I$ characteristics of $\mathrm{Nb}-\mathrm{Ti}$ and $\mathrm{Nb}_{3} \mathrm{Sn}$ wires using a normal shunt to make $d^{2} V / d I^{2}$ analysis possible [10]-[13]. We should note that the shunt resistance of the normal metal matrices of $\mathrm{Cu}$ in $\mathrm{Nb}-\mathrm{Ti}$ and $\mathrm{Ag}$ in $\mathrm{Bi}-2212$ are rather similar, both having residual resistivity ratio (RRR) > 100 [14], [15].

\section{EXPERIMENTAL}

Two Bi-2212 RWs with the same nominal powder composition of $\mathrm{Bi}_{2.17} \mathrm{Sr}_{1.94} \mathrm{Ca}_{0.90} \mathrm{Cu}_{1.98} \mathrm{O}_{\mathrm{x}}$ but very different powders and time of fabrication were selected. The pmm100913 and pmm180410 wires were fabricated in 2010 and 2018 by Bruker-Oxford Superconducting Technology (B-OST) using Nexans granulate (lot 77) and Engi-Mat (LXB 116) powder. Both powders were state-of-the-art at the time. Double restack multifilamentary wires were made by the Powder-In-Tube (PIT) process using powder-filled pure Ag tubes and an outer sheath of $\mathrm{Ag}-0.2 \mathrm{wt} . \% \mathrm{Mg}$ alloy. The heat treatment of $1.5 \mathrm{~m}$ spiral wires was done at 50 bar over-pressure with an oxygen partial pressure of 1 bar [4]. Wires were then soldered onto a brass ITER-like barrel [16] and the $V-I$ characteristics of multiple $10 \mathrm{~cm}$ sections of each sample were measured using standard four-probe transport measurement methods at $4.2 \mathrm{~K}$ in magnetic fields up to $15 \mathrm{~T}$. The peak applied currents were well above the standard $1 \mu \mathrm{V} \cdot \mathrm{cm}^{-1}$ criterion limit far into the flux flow regime, so as to obtain the full $I_{\mathrm{c}}$ distribution. A standard $\mathrm{Nb}-47 \mathrm{wt} . \% \mathrm{Ti}$ wire of high $J_{\mathrm{c}}$ manufactured by Supercon Inc. was also measured. Detailed specifications of the samples are listed in Table I.

\section{A. Measurements of the Critical Current Distribution}

Traditional four-probe transport measurements reveal only the lower end of the $I_{c}$ distribution. In a well-optimized material like $\mathrm{Nb}-\mathrm{Ti}$ where current is believed to flow uniformly, magnetization and transport measurements typically agree well when corrections for electric field $(E)$ differences are made (and filament sausaging is avoided). Bi-2212 is more complex: the current path is percolative and uncertain with a fractional occupancy of as little as $1 \%$ [6]. Moreover, most transport measurements typically characterize only $\sim 1$ $\mathrm{cm}$ of conductor due to use of straight wires in narrow-bore magnets. Because of the greater stability of Bi-2212 compared to $\mathrm{Nb}-\mathrm{Ti}$ and $\mathrm{Nb}_{3} \mathrm{Sn}$ it appears that a relatively high criterion of $1 \mu \mathrm{V} \cdot \mathrm{cm}^{-1}$ reliably predicts the quench performance of even potted magnets [17], making it interesting to explore the $V-I$ transition at both lower and higher $E$ values.

An early method that was successfully used to characterize the $I_{\mathrm{c}}$ distribution of $\mathrm{Nb}-\mathrm{Ti}$ and $\mathrm{Nb}_{3} \mathrm{Sn}$ wires used a substantial normal metal shunt to avoid conductor burn out [11], [18]. The method models the $I_{\mathrm{c}}$ distribution as a series array of many short longitudinal sections of varying $I_{\mathrm{c}}$ [13], [19]. As the applied current exceeds the local critical current $\left(i_{\mathrm{c}}\right)$ of a section, the section transitions from a pinned to a flux flow state. Since the flux flow resistivity is two to three orders of magnitude higher than the silver matrix and the high conductance barrel [11], almost all the excess current is carried by the normal matrix and shunt without excessive heating and depinning of vortices in the superconductor. As originally formulated by Baixeras and Fournet [10], the voltage across a sub-element with critical current $i_{\mathrm{c}}$ as a function of applied current $I$ is given by a series distribution of variable $i_{\mathrm{c}}$ elements:

$$
V\left(I, i_{\mathrm{c}}\right)=R\left(I-i_{\mathrm{c}}\right)
$$

The normal method of degradation of $I_{\mathrm{c}}$ in $\mathrm{Nb}-\mathrm{Ti}$ is by onset of filament sausaging which fits the series $i_{\mathrm{c}}$ array model well but almost all other practical superconductors have more complex series-parallel current paths. Plummer and Evetts [18] extended this analysis to filamentary $\mathrm{Nb}_{3} \mathrm{Sn}$ conductors, where the normal bronze matrix can have higher ohmic dissipation than flux flow in the $\mathrm{Nb}_{3} \mathrm{Sn}$ filaments (bronze $\mathrm{Cu}$ is always poisoned by the residual $\mathrm{Sn}$ content, typically $0.15-1$ at.\% meaning that its RRR is $<5$ ), using a dynamic flux flow relation to determine the $\mathrm{Nb}_{3}$ Sn distribution, arriving at the same equation as Baixeras and Fournet. In their model, the $I_{\mathrm{c}}$ distribution was assumed to be Gaussian due to many variations of grain size, local Sn content and filament uniformity, none of which were well controlled or easy to measure. In this more general case of a wire with many filaments, varying vortex pinning and varying active cross-section, the distribution of $i_{\mathrm{c}}$ values is both parallel and series and complex. Denoting the desired distribution $\phi\left(i_{\mathrm{c}}\right)$ as the probability distribution density of $i_{\mathrm{c}}$ in the wire suggests use of a Gaussian function since many independent factors are locally determining the local $i_{\mathrm{c}}$ values. Taking $R_{\mathrm{eff}}$ as the effective resistance of the normal currents in the stabilizer and shunt, integrating over all possible $i_{\mathrm{c}}$ values gives the following expression for the total voltage measured across the wire at a given applied current $I$ :

$$
\begin{gathered}
V(I)=R_{\mathrm{eff}} \int_{0}^{I}\left(I-i_{\mathrm{c}}\right) \phi\left(i_{\mathrm{c}}\right) d i_{\mathrm{c}} \\
\phi(I)=\frac{1}{\sqrt{2 \pi \sigma^{2}}} \exp \frac{-(I-\mu)^{2}}{2 \sigma^{2}} \\
\frac{d^{2} V(I)}{d I^{2}}=R_{\mathrm{eff}} \phi(I)=\frac{R_{\mathrm{eff}}}{\sqrt{2 \pi \sigma^{2}}} \exp \frac{-(I-\mu)^{2}}{2 \sigma^{2}}
\end{gathered}
$$


Differentiating $V(I)$ twice with respect to $I$ yields $R_{\text {eff }} \cdot \phi(I)$, which allows extraction of the $I_{\mathrm{c}}$ distribution and $R_{\text {eff. }}$. To reduce noise, a Savitzky-Golay filter was used to smooth the data before calculating the second derivative [11], [20] by regression fitting a second order polynomial to a typical comb size $(m)$ of $7-15 V-I$ points, the analytical derivative being determined from the coefficient at the center of a group of $2 m+1$ points. The $7-15$ point comb size was kept low to avoid smoothing out subtle features of the distribution curve shape. Since the factors that affect local critical current are thought to be randomly distributed and uncorrelated, the distribution of $i_{\mathrm{c}}$ plausibly converges to a Gaussian as predicted by the central limit theorem [13], [18], [21], [22] enabling extraction of distribution parameters, such as the mean $I_{\mathrm{c}}(\mu$ in (4)) and the standard deviation $(\sigma)$.

\section{B. The Fraction of Superconductor in Flux Flow}

$f_{\mathrm{D}}(I)$ represents the fraction of superconductor wire in the flux flow state at a given current and this was calculated from $d^{2} V / d I^{2}$ at $I_{\mathrm{c}}$ determined by both $0.1 \mu \mathrm{V} \cdot \mathrm{cm}^{-1}$ and $1 \mu \mathrm{V} \cdot \mathrm{cm}^{-1}$ criteria according to:

$$
f_{\mathrm{D}}(I)=\int_{0}^{I} \phi\left(i_{\mathrm{c}}\right) d i_{\mathrm{c}}
$$

\section{Critical Current Measurements of Short $(4.5 \mathrm{~cm})$ Samples}

To compare regular measurements on short samples to those determined on the shunted barrel samples, $10 \mathrm{~cm}$ long wires of Bi-2212 were heat treated along with each spiral sample. Transport critical currents $I_{\mathrm{c}}$ were measured using the fourprobe method at $4.2 \mathrm{~K}$ in perpendicular magnetic fields up to $15 \mathrm{~T}$ using both $0.1 \mu \mathrm{V} \cdot \mathrm{cm}^{-1}$ and $1 \mu \mathrm{V} \cdot \mathrm{cm}^{-1}$ criteria. The resistive transition index ( $n$ value) was calculated by fitting the $V-I$ curve from $0.6-20 \mu \mathrm{V} \cdot \mathrm{cm}^{-1}$.

\section{Results}

Fig. 1(a) shows the $I_{\mathrm{c}}$ distribution of a $10 \mathrm{~cm}$ section of the higher- $J_{\mathrm{c}}$ Engi-Mat wire at $5 \mathrm{~T}$ and $14 \mathrm{~T}$. The basic features are similar in all measured samples. Both $5 \mathrm{~T}$ and $14 \mathrm{~T}$ curves are slightly asymmetric due to an extended high current tail but it is also evident that the $14 \mathrm{~T} I_{\mathrm{c}}$ distribution is sharper than the $5 \mathrm{~T}$ distribution. Two separate lines mark the short sample $I_{\mathrm{c}}$ position based on $0.1 \mu \mathrm{V} \cdot \mathrm{cm}^{-1}$ and $1 \mu \mathrm{V} \cdot \mathrm{cm}^{-1}$ criteria. The $I_{\mathrm{c}}$ of the higher- $J_{\mathrm{c}}$ Engi-Mat short sample at $1 \mu \mathrm{V} \cdot \mathrm{cm}^{-1}$ is $1078 \mathrm{~A}$ at $5 \mathrm{~T}$ and $782 \mathrm{~A}$ at $14 \mathrm{~T}$ compared to the distribution analysis mean $I_{\mathrm{c}} \sim 1039.8 \pm 16.9 \mathrm{~A}, \sigma \sim 99.3 \pm 4.9 \mathrm{~A}$, and $\sigma / \mu \sim 0.095 \pm 0.0044$ at $5 \mathrm{~T}$. By contrast, the older and lower$J_{\mathrm{c}}$ Nexans wire shows mean $I_{\mathrm{c}} \sim 448.2 \pm 5.1 \mathrm{~A}, \sigma \sim 59.1 \pm$ $3.4 \mathrm{~A}$, and $\sigma / \mu \sim 0.132 \pm 0.0084$ at $5 \mathrm{~T}$. For comparison to a more classical wire Fig. 1(b) shows the $I_{\mathrm{c}}$ distribution of the Supercon $\mathrm{Nb}-\mathrm{Ti}$ wire at various fields. At $5 \mathrm{~T}$, it has $I_{\mathrm{c}} \sim$ $394.9 \pm 3.6 \mathrm{~A}, \sigma \sim 18.6 \pm 1.5 \mathrm{~A}$, and $\sigma / \mu \sim 0.047 \pm 0.0034$. $\mathrm{The} \mathrm{Nb}-\mathrm{Ti}$ wire distribution width is significantly lower than both Bi-2212 samples at fields well below $H_{\text {irr }}$ but does cross the Engi-Mat Bi-2212 wire close to $H_{\text {irr }}$. Further details of the distribution parameters are listed in Table II.
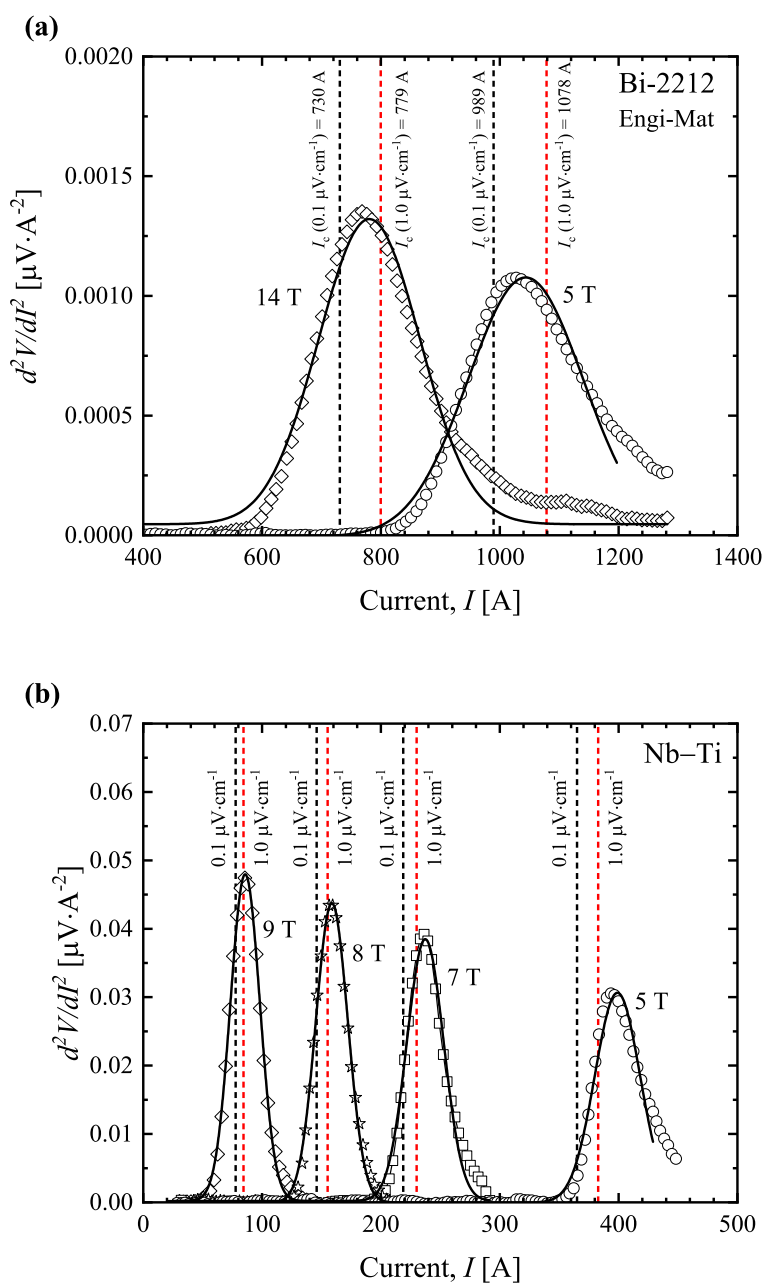

Fig. 1. Critical current distribution of (a) pmm180410 (Engi-Mat) wire at $5 \mathrm{~T}$ (circle) and $14 \mathrm{~T}$ (diamond) at $4.2 \mathrm{~K}$, (b) $\mathrm{Nb}-\mathrm{Ti}$ wire at $5 \mathrm{~T}$ (circle), $7 \mathrm{~T}$ (square), $8 \mathrm{~T}$ (star), and $9 \mathrm{~T}$ (diamond) at $4.2 \mathrm{~K}$. Two separate lines are drawn in the distribution plot for each field to delineate the short sample $I_{\mathrm{c}}$ based on $0.1 \mu \mathrm{V} \cdot \mathrm{cm}^{-1}$ (black dotted line) and $1 \mu \mathrm{V} \cdot \mathrm{cm}^{-1}$ (red dotted line) criterion. Solid black lines represents the Gaussian distribution.

Fig. 2(a) shows $J_{\mathrm{c}}(H)$ for Engi-Mat, Nexans and $\mathrm{Nb}-\mathrm{Ti}$ wires. The power law fit of $J_{\mathrm{c}}(H)$ from $3 \mathrm{~T}$ to $15 \mathrm{~T}$ for both Bi-2212 wires is shown in the inset of Fig. 2(a).

Fig. 2(b) shows $\sigma / \mu$ of the $I_{\mathrm{c}}$ distribution for Engi-Mat, Nexans, and $\mathrm{Nb}-\mathrm{Ti}$ wires with error bars of one standard deviation calculated from multiple sections of the spiral on each barrel. For $\mathrm{Nb}-\mathrm{Ti}, \sigma / \mu$ increases monotonically with field from $0.034 \pm 0.0023$ at $3 \mathrm{~T}$ to $0.125 \pm 0.0272$ at $10 \mathrm{~T}$, but it varies only slowly with increasing magnetic field in Bi-2212 wires, most likely because, unlike for $\mathrm{Nb}-\mathrm{Ti}, H / H_{\text {irr }}$ is always significantly lower than unity. At $10 \mathrm{~T}$, Nexans and Engi-Mat wires show $\sigma / \mu \sim 0.14$, and 0.10 , respectively, but is then 0.13 for $\mathrm{Nb}-\mathrm{Ti}$. The fractional energy dissipation $\left(f_{\mathrm{D}}\right)$ and the ratio of short sample critical current to mean critical current $\left(I_{\mathrm{c}} / \mu\right)$ are listed in Table II. The $f_{\mathrm{D}}\left(I_{\mathrm{c}}\right)$ of the Engi-Mat wire, where $I_{\mathrm{c}}$ using the $1 \mu \mathrm{V} \cdot \mathrm{cm}^{-1}$ electric field criterion on the $4.5 \mathrm{~cm}$ long sample shows that $60-65 \%$ of the superconductor has transitioned from the flux pinning 
(a)

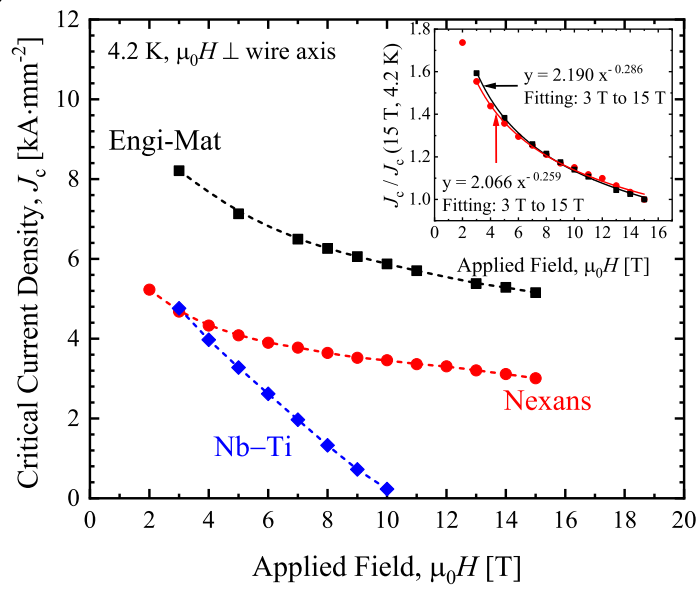

(b)

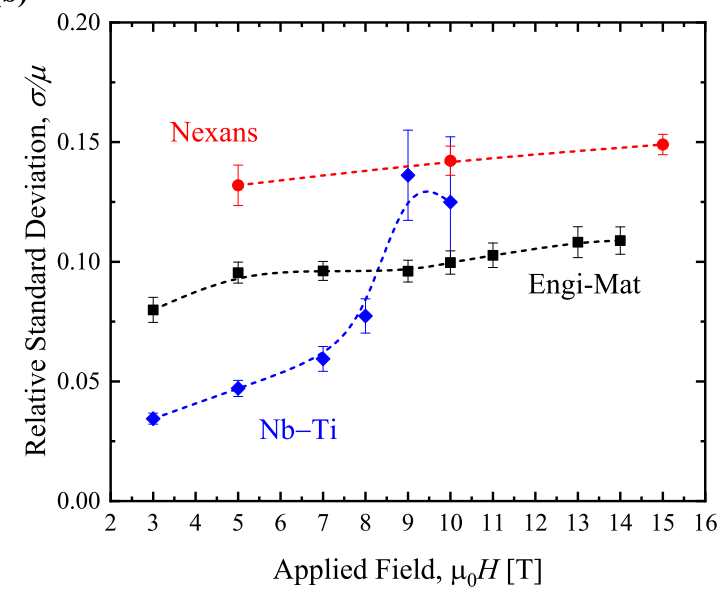

Fig. 2. (a) Critical current density evaluated at $1 \mu \mathrm{V} \cdot \mathrm{cm}^{-1}$, and (b) relative standard deviation of $I_{\mathrm{c}}$ distribution as a function of applied field for pmm180410 (square), pmm100913 (circle), and $\mathrm{Nb}-\mathrm{Ti}$ (diamond) wires at $4.2 \mathrm{~K}$. The power law fit from $3 \mathrm{~T}$ to $15 \mathrm{~T}$ for both $\mathrm{Bi}-2212$ wire is shown in the inset. Dashed lines are guides for the eye.

to the flux flow state. In contrast, the more conservative $0.1 \mu \mathrm{V} \cdot \mathrm{cm}^{-1}$ criterion corresponds to $22-27 \%$ in flux flow. In the lower- $J_{\mathrm{c}}$ Nexans wire $f_{\mathrm{D}}$ ranges from $43-50 \%$ at $I_{\mathrm{c}}\left(1 \mu \mathrm{V} \cdot \mathrm{cm}^{-1}\right)$, while the conservative $\left(I_{\mathrm{c}} 0.1 \mu \mathrm{V} \cdot \mathrm{cm}^{-1}\right)$ criterion yields $\sim 15-19 \%$. For the $\mathrm{Nb}-\mathrm{Ti}$ wire, $f_{\mathrm{D}}$ increased from $14 \%$ to $69 \%$ with an increasing magnetic field. The $I_{\mathrm{c}} / \mu$ ratio of Engi-Mat, Nexans, and $\mathrm{Nb}-\mathrm{Ti}$ wires are $\sim 1.04, \sim$ $0.98-1.0$, and $0.94-1.07$, respectively.

\section{Discussion}

In this work, we have compared the $I_{c}$ distribution of two Bi-2212 RWs made by B-OST prepared with newer and better and older and worse quality powders from EngiMat and Nexans. We were motivated to better understand the conclusion of Brown et al. [6] that the magnitude of $J_{\mathrm{c}}$ in $\mathrm{Bi}-2212$ wires was almost completely determined by the effective connectivity of the current path which we interpreted to be very small since the ratio $J_{\mathrm{c}} / J_{\mathrm{d}}$ is of order $1 \%$ or less. Even if the vortex pinning in Bi-2212 is weak (it is also not yet clarified and may just be due to cation defect fluctuations within the Bi-2212 structure), such a low ratio of $J_{\mathrm{c}}$ to $J_{\mathrm{d}}$ implies that the long range current path may occupy a small fraction of the total cross-section. In the absence of detailed measurements of $J_{\mathrm{c}}$ at the filament level (we have done this earlier for sections of Bi-2223 filament and found values of $J_{c}$ several times the average [23]), we started this work to see if the distribution of $J_{\mathrm{c}}$ values extracted from $d^{2} V / d I^{2}$ measurements would offer a better understanding of the differences between representative wires made with the former champion Nexans and the more recent champion EngiMat powders with about a factor of 2 difference in the filament $J_{\mathrm{c}}$ defined by the measurement of $I_{\mathrm{c}}$ divided by the fully densified Bi-2212 cross-section.

In analyzing the data we take the common view [12], [21], [22], [24] that the local $I_{\mathrm{c}}$ along the wire is controlled by multiple independent factors that justify a Gaussian approximation consistent with the central limit theorem [18]. Gaussian distributions have earlier been measured in $\mathrm{Nb}_{3} \mathrm{Sn}$ [13], [18], [19] with $\sigma / \mu$ values of 0.15 at $15 \mathrm{~T}$, about $0.6 H_{\text {irr }}$. Here we show that a high- $J_{\mathrm{c}} \mathrm{Nb}-\mathrm{Ti}$ has a significantly smaller $\sigma / \mu$ of $\sim 0.05$ at $5 \mathrm{~T}\left(\sim 0.5 H_{\text {irr }}\right)$, although it does rise significantly above 0.1 at $8 \mathrm{~T}$ close to $H_{\text {irr }}$. Both $\mathrm{Nb}-\mathrm{Ti}$ and $\mathrm{Nb}_{3} \mathrm{Sn}$ are isotropic with respect both to vortex pinning and to $H_{\mathrm{c} 2}$ [25], [26]. Given the meandering $c$ - axis along the filament, we expect that some grains will have very favorable orientations for $H_{\mathrm{c} 2}$ and some quite unfavorable. Not yet being clear what the strong pinning centers are in Bi-2212, we cannot yet $a$ priori say whether the high $H_{\mathrm{c} 2} a b$ - plane orientation has stronger pinning than the $c$ - axis orientation but we might expect that the $d^{2} V / d I^{2}$ would be broadened on the high $I_{\mathrm{c}}$ side by this anisotropy.

Indeed, we found that our two Bi-2212 wires showed Gaussian behavior over about three quarters of their $I_{\mathrm{c}}$ distribution but also possessed a notable non-Gaussian extended tail beyond about $150 \%$ of the mean $I_{c}$. Such a tail was also seen in studies of Bi-2223 tapes [27]. Since both Bi-2223 and Bi-2212 are strongly anisotropic cuprates and both have percolative current paths, broader transitions and some nonGaussian behavior is not unexpected, particularly at higher $I_{\mathrm{c}}$ values that may correspond to grains with higher $H_{\mathrm{c} 2}$ values. Qualitatively then the non-Gaussian high-side tail has a plausible physical basis, even if we are very far from being able to access the whole range of $i_{c}$ of grains with strongly varying orientations.

It is worth pointing out that the $\mathrm{Bi}-2212$ distributions yield mean critical current values $\mu$ within a few percent of the measured short $(\sim 5 \mathrm{~cm})$ sample $I_{\mathrm{c}}\left(1 \mu \mathrm{V} \cdot \mathrm{cm}^{-1}\right)$ results, with a dissipation fraction $f_{\mathrm{D}}$ rather independent of field, whereas for $\mathrm{Nb}-\mathrm{Ti} f_{\mathrm{D}}$ increases from $15 \%$ to $68 \%$ between $3 \mathrm{~T}$ and $10 \mathrm{~T}$ with $\mu$ moving from above to below $I_{\mathrm{c}}$. This corresponds to a decrease in dissipation from $\sim 200 \mathrm{~mW} \cdot \mathrm{cm}^{-3}$ at $3 \mathrm{~T}$ to only $10 \mathrm{~mW} \cdot \mathrm{cm}^{-3}$ at $10 \mathrm{~T}$ at a $1 \mu \mathrm{V} \cdot \mathrm{cm}^{-1}$ criterion, in the context of the low enthalpy margin of $\mathrm{Nb}-\mathrm{Ti}$, on the order of $1-$ $10 \mathrm{~mJ} \cdot \mathrm{cm}^{-3}$. The higher dissipation occurring at lower fields causes LTS conductors to quench rapidly when even a fraction of the distribution is normal, as seen by the higher $n$ values of their transitions. By contrast, for Bi-2212, the dissipation 
TABLE II

Parameters Derived From The Critical Current Distribution

\begin{tabular}{|c|c|c|c|c|c|c|c|c|c|c|}
\hline \multirow[t]{2}{*}{ Sample $^{\dagger}$} & $\begin{array}{l}\text { Magnetic } \\
\text { Field }\end{array}$ & Mean, $\mu$ & $\begin{array}{l}\text { Standard } \\
\text { Deviation, } \sigma\end{array}$ & $\begin{array}{l}\text { Relative Std. } \\
\text { Deviation, } \sigma / \mu\end{array}$ & $R_{\mathrm{eff}^{+}}$ & $\begin{array}{l}\text { Critical } \\
\text { Current, } I_{\mathrm{c}}{ }^{++}\end{array}$ & $n$ value & $f_{D}\left(I_{\mathrm{c}}\right)^{\mathrm{a}}$ & $f_{\mathrm{D}}\left(I_{\mathrm{C}}\right)^{\mathrm{b}}$ & $\frac{I_{c}}{\mu}$ \\
\hline & {$[\mathrm{T}]$} & [A] & {$[\mathrm{A}]$} & & {$\left[\mu \Omega \cdot \mathrm{cm}^{-1}\right]$} & {$[\mathrm{A}]$} & & {$[\%]$} & {$[\%]$} & \\
\hline pmm180410 & 3 & 1210.6 & 96.6 & 0.080 & 0.023 & 1242.5 & 26.6 & 22.8 & 61.9 & 1.03 \\
\hline \multirow[t]{7}{*}{ (Engi-Mat) } & 5 & 1039.8 & 99.3 & 0.095 & 0.028 & 1078.8 & 26.7 & 30.5 & 64.2 & 1.04 \\
\hline & 7 & 947.6 & 91.2 & 0.096 & 0.027 & 982.6 & 25.6 & 28.7 & 64.1 & 1.04 \\
\hline & 9 & 881.3 & 84.4 & 0.096 & 0.027 & 916.5 & 26.2 & 29.6 & 63.7 & 1.04 \\
\hline & 10 & 859.6 & 85.7 & 0.100 & 0.028 & 888.9 & 25.7 & 29.5 & 63.1 & 1.03 \\
\hline & 11 & 834.0 & 85.7 & 0.103 & 0.028 & 863.3 & 25.2 & 29.1 & 62.6 & 1.04 \\
\hline & 13 & 793.8 & 85.9 & 0.108 & 0.029 & 814.1 & 25.0 & 24.9 & 60.9 & 1.03 \\
\hline & 14 & 774.2 & 84.3 & 0.109 & 0.029 & 799.7 & 22.2 & 30.6 & 62.1 & 1.03 \\
\hline pmm100913 & 5 & 448.2 & 59.1 & 0.132 & 0.030 & 445.2 & 17.4 & 16.5 & 49.6 & 0.99 \\
\hline \multirow[t]{2}{*}{ (Nexans) } & 10 & 376.6 & 53.5 & 0.142 & 0.032 & 377.2 & 18.8 & 18.6 & 47.8 & 1.00 \\
\hline & 15 & 336.2 & 50.1 & 0.149 & 0.032 & 328.0 & 15.2 & 13.3 & 43.8 & 0.98 \\
\hline \multirow[t]{6}{*}{$\mathrm{Nb}-\mathrm{Ti}$} & 3 & 589.7 & 20.3 & 0.034 & 0.047 & 556.1 & 50.2 & 1.7 & 14.3 & 0.94 \\
\hline & 5 & 394.9 & 18.6 & 0.047 & 0.048 & 382.8 & 62.6 & 8.1 & 30.5 & 0.97 \\
\hline & 7 & 233.0 & 13.8 & 0.059 & 0.048 & 230.1 & 58.6 & 18.6 & 43.9 & 0.99 \\
\hline & 8 & 154.9 & 12.0 & 0.077 & 0.048 & 155.2 & 55.2 & 23.6 & 53.5 & 1.00 \\
\hline & 9 & 82.1 & 11.2 & 0.136 & 0.048 & 84.4 & 44.5 & 36.1 & 59.9 & 1.03 \\
\hline & 10 & 25.1 & 3.1 & 0.125 & 0.049 & 26.9 & 15.8 & 21.3 & 68.5 & 1.07 \\
\hline
\end{tabular}

${ }^{\dagger}$ Distribution is measured for multiple sections of the spiral sample at each field. Here average results of the fitting parameters are listed.

${ }^{+}$Each voltage tap in pmm180410, pmm100913, and $\mathrm{Nb}-$ Ti samples is $10 \mathrm{~cm}, 20 \mathrm{~cm}$, and $30 \mathrm{~cm}$ apart, respectively.

${ }^{++}$Critical current is calculated from a $4.5 \mathrm{~cm}$ short sample based on $1 \mu \mathrm{V} \cdot \mathrm{cm}^{-1}$ criterion at $4.2 \mathrm{~K}$.

${ }_{\mathrm{b}}^{\mathrm{a}} f_{\mathrm{D}}\left(I_{\mathrm{c}}\right)$ is calculated based on short sample $I_{\mathrm{c}}$ at $0.1 \mu \mathrm{V} \cdot \mathrm{cm}^{-1}$ criterion.

${ }^{\mathrm{b}} f_{\mathrm{D}}\left(I_{\mathrm{c}}\right)$ is calculated based on short sample $I_{\mathrm{c}}$ at $1 \mu \mathrm{V} \cdot \mathrm{cm}^{-1}$ criterion.

changes are much less, $\sim 160-100 \mathrm{~mW} \cdot \mathrm{cm}^{-3}$ from $3-$ $14 \mathrm{~T}$ for the Engi-Mat wire, and the enthalpy margin is two orders of magnitude larger, leading to more uniform $f_{\mathrm{D}}$ and $n$ values. At higher fields the margin decays only gradually for Bi-2212, which still has over 10 times more margin at $30 \mathrm{~T}$ than $\mathrm{Nb}-\mathrm{Ti}$ has at $5 \mathrm{~T}$.

The relative standard deviation $(\sigma / \mu)$ is a significant parameter which considers both the breadth and the mean of the $I_{\mathrm{c}}$ distribution. Previous investigations of the $I_{\mathrm{c}}$ distribution of technological conductors yielded $\sigma / \mu \sim 0.02-0.50$ for $\mathrm{Nb}_{3} \mathrm{Sn}$, $\mathrm{Nb}-\mathrm{Ti}$, and $\mathrm{Bi}-2223$ [11], [13]. However, Warnes et al. also reported an enhanced $\sigma / \mu \sim 0.14$ for a severely sausaged $\mathrm{Nb}-\mathrm{Ti}$ wire at $4 \mathrm{~T}$ (reduced field, $H / H_{\text {irr }} \sim 0.36$ ) which increased to $\sim 0.33$ at $10 \mathrm{~T}$ [11], thus correlates a high $\sigma / \mu$ value with strong filament sausaging. Here we measured the $I_{\mathrm{c}}$ distributions of $\mathrm{Bi}-2212$ wires up to $15 \mathrm{~T}$ but since this is only $10-15 \%$ of $H_{\text {irr }}$, the complete $H / H_{\text {irr }}$ dependence of $\sigma / \mu$ in our wires is not known. Hence, we believe that it is more appropriate to compare the low field $\sigma / \mu$ values of $\mathrm{Nb}-\mathrm{Ti}$ to our Bi-2212 wires. The relative broadening width near the irreversibility field seen in the $\mathrm{Nb}-\mathrm{Ti}$ probably results from large fluctuations in the effective vortex pinning near $H_{\text {irr }}$ as the elementary pinning forces generated by the dense $\alpha$-Ti vortex-pins become proximity coupled to the superconducting $\beta$-phase matrix [28].

In line with our original conjecture, we did find a marked difference in $\sigma / \mu$ between the Engi-Mat (pmm180410) and Nexans (pmm100913) wires. It is evident (see Fig. 2(b)) that the older and lower- $J_{\mathrm{c}}$ Nexans wire has about $40 \%$ higher $\sigma / \mu$ $(\sim 0.1$ vs. 0.14). As noted earlier by Brown et al. [6], many wires made with Nexans, Engi-Mat and other powder sets had the same normalized $J_{\mathrm{c}}(H)$ characteristics, even though the magnitude of $J_{\mathrm{c}}$ varied by almost 6 . We conclude that the effective percolative connectivity of wires is what is really controlling the $J_{\mathrm{c}}$ magnitude. The modern fine particle EngiMat powder has more uniform characteristics than the earlier generation Nexans powder as demonstrated experimentally by Jiang et al. [4]. We conclude that much of the $\sim 60 \% J_{\mathrm{c}}$ improvement is due to improved filament connectivity of the Engi-Mat powder [4]. Thus, we conclude that the $30 \%$ lower $\sigma / \mu$ in our Engi-Mat sample compared to the lower- $J_{\mathrm{c}}$ Nexans sample is due to improved connectivity associated with the much finer and more uniform Engi-Mat powder.

As noted above, the Nexans $\sigma / \mu$ values are very similar to that of a severely sausaged $\mathrm{Nb}-\mathrm{Ti}$ wire [11]. Knowing that Nexans powder has many hard particles of varying size which distort the filament structure, it is plausible to compare the degraded connectivity of Bi-2212 wire made with Nexans powder to sausaged $\mathrm{Nb}-\mathrm{Ti}$ wire [11]. However, we do not conclude that filament sausaging is the sole reason for the degraded $\sigma / \mu$ of our Nexans sample. It is interesting that a $\mathrm{Bi}-2223$ wire $(\mu \sim 143.6 \mathrm{~A}, \sigma \sim 16.8 \mathrm{~A}, \sigma / \mu \sim 0.12$ at $4.2 \mathrm{~K}$, $0.5 \mathrm{~T})$ also had a large $\sigma / \mu$ [27]. Many other factors are also still in play, one being degraded grain-to-grain connectivity that is certainly present in both $\mathrm{Bi}-2212$ and $\mathrm{Bi}-2223$.

Although better connected than the Nexans powder wire, the higher- $J_{\mathrm{c}}$ Engi-Mat powder wire has a $\sim 3$ fold higher $\sigma / \mu$, and an almost $50 \%$ smaller $n$ value compared to our high- $J_{c}$ $\mathrm{Nb}-\mathrm{Ti}$ wire. Based on the experimental evidence it can be expected that $I_{\mathrm{c}}$ of Bi-2212 RWs could be increased further if the $\sigma / \mu$ can be minimized by identifying and addressing connectivity limitations. To this end, we believe that the $\sigma / \mu$ 
value can be used as a quantitative parameter for conductor quality control.

\section{Conclusion}

In fact, the $I_{\mathrm{c}}$ distribution, though wider for Bi-2212 than for optimized $\mathrm{Nb}-47$ wt.\% $\mathrm{Ti}$, is not a great deal larger, in spite of Bi-2212 being strongly anisotropic along each filament, possessing current-blocking regions, and having highly nonuniform filament shapes. The much lower- $J_{\mathrm{c}}$ Nexans wire has the same normalized vortex pinning (same normalized $J_{\mathrm{c}}(H)$ curve) and a broader $\sigma / \mu$, suggestive of poorer connectivity. The relative standard deviation of Engi-Mat wire is 30\% lower than Nexans wire. The implication is that effective filament connectivity can be quantified from the $d^{2} V / d I^{2}$ measurement. We believe that measurements like these can be a useful tool in further understanding of how best to raise $J_{\mathrm{c}}$ in Bi-2212 conductors.

\section{ACKNOWLEDGMENT}

We acknowledge the help of G. Bradford, V. S. Griffin, and E. Miller of the National High Magnetic Field Laboratory.

\section{REFERENCES}

[1] D. C. Larbalestier, J. Jiang, U. P. Trociewitz, F. Kametani, C. Scheuerlein, M. Dalban-Canassy, M. Matras, P. Chen, N. C. Craig, P. J. Lee, and E. E. Hellstrom, "Isotropic round-wire multifilament cuprate superconductor for generation of magnetic fields above 30 T," Nature Materials, vol. 13, no. 4, pp. 375-381, 2014.

[2] H. Miao, Y. Huang, S. Hong, and J. A. Parrell, "Recent advances in Bi-2212 round wire performance for high field applications," IEEE Transactions on Applied Superconductivity, vol. 23, no. 3, p. 6400104, 2012.

[3] J. Parrell, M. Field, Y. Zhang, and S. Hong, " $\mathrm{Nb}_{3}$ Sn conductor development for fusion and particle accelerator applications," AIP Conference Proceedings, vol. 711, no. 1, pp. 369-375, 2004.

[4] J. Jiang, G. Bradford, S. I. Hossain, M. D. Brown, J. Cooper, E. Miller, Y. Huang, H. Miao, J. A. Parrell, M. White et al., "High-performance Bi-2212 round wires made with recent powders," IEEE Transactions on Applied Superconductivity, vol. 29, no. 5, pp. 1-5, 2019.

[5] V. Kopylov, A. Koshelev, I. Schegolev, and T. Togonidze, "The role of surface effects in magnetization of high- $T_{\mathrm{c}}$ superconductors," Physica C: Superconductivity, vol. 170, no. 3-4, pp. 291-297, 1990.

[6] M. D. Brown, J. Jiang, C. Tarantini, D. Abraimov, G. Bradford, J. Jaroszynski, E. E. Hellstrom, and D. C. Larbalestier, "Prediction of the $J_{\mathrm{c}}(B)$ behavior of Bi-2212 wires at high field," IEEE Transactions on Applied Superconductivity, vol. 29, no. 5, pp. 1-4, 2019.

[7] F. Kametani, J. Jiang, M. Matras, D. Abraimov, E. Hellstrom, and D. Larbalestier, "Comparison of growth texture in round Bi2212 and flat Bi2223 wires and its relation to high critical current density development," Scientific Reports, vol. 5, p. 8285, 2015.

[8] T. Shen, J. Jiang, F. Kametani, U. P. Trociewitz, D. C. Larbalestier, and E. E. Hellstrom, "Heat treatment control of $\mathrm{Ag}-\mathrm{Bi}_{2} \mathrm{Sr}_{2} \mathrm{CaCu}_{2} \mathrm{O}_{x}$ multifilamentary round wire: investigation of time in the melt," Superconductor Science and Technology, vol. 24, no. 11, p. 115009, 2011.

[9] J. Jiang, A. Francis, R. Alicea, M. Matras, F. Kametani, U. P. Trociewitz, E. E. Hellstrom, and D. C. Larbalestier, "Effects of filament size on critical current density in overpressure processed Bi-2212 round wire," IEEE Transactions on Applied Superconductivity, vol. 27, no. 4, pp. 1-4, 2016.

[10] J. Baixeras and G. Fournet, "Vortex displacement losses in a non-ideal type II superconductor," Journal of Physics and Chemistry of Solids, vol. 28 , no. 8, pp. 1541-1547, 1967.

[11] W. Warnes and D. Larbalestier, "Critical current distributions in superconducting composites," Cryogenics, vol. 26, no. 12, pp. 643-653, 1986.

[12] W. H. Warnes, "A model for the resistive critical current transition in composite superconductors," Journal of Applied Physics, vol. 63, no. 5, pp. 1651-1662, 1988.
[13] H. Mueller, F. Hornung, A. Rimikis, and T. Schneider, "Critical current distribution in composite superconductors," IEEE Transactions on Applied Superconductivity, vol. 17, no. 2, pp. 3757-3760, 2007.

[14] P. Li, L. Ye, J. Jiang, and T. Shen, "RRR and thermal conductivity of Ag and $\mathrm{Ag}-0.2$ wt.\% Mg alloy in $\mathrm{Ag} / \mathrm{Bi}-2212$ wires," in IOP Conference Series: Materials Science and Engineering, vol. 102, no. 1. IOP Publishing, 2015, p. 012027.

[15] M. Bonura, F. Avitabile, C. Barth, J. Jiang, D. Larbalestier, A. Fête, A. Leo, L. Bottura, and C. Senatore, "Very-high thermal and electrical conductivity in overpressure-processed $\mathrm{Bi}_{2} \mathrm{Sr}_{2} \mathrm{CaCu}_{2} \mathrm{O}_{8+\mathrm{x}}$ wires," Materials Research Express, vol. 5, no. 5, p. 056001, 2018.

[16] P. Bruzzone, H. H. ten Kate, M. Nishi, A. Shikov, J. Minervini, and M. Takayasu, "Bench mark testing of $\mathrm{Nb}_{3} \mathrm{Sn}$ strands for the ITER model coil," in Advances in Cryogenic Engineering Materials, L. T. Summers, Ed. Boston, MA: Springer, US, 1996, pp. 1351-1358.

[17] T. Shen, E. Bosque, D. Davis, J. Jiang, M. White, K. Zhang, H. Higley, M. Turqueti, Y. Huang, H. Miao et al., "Stable, predictable and trainingfree operation of superconducting $\mathrm{Bi}-2212$ rutherford cable racetrack coils at the wire current density of $1000 \mathrm{~A} / \mathrm{mm}^{2}$," Scientific Reports, vol. 9, no. 1, pp. 1-9, 2019.

[18] C. Plummer and J. Evetts, "Dependence of the shape of the resistive transition on composite inhomogeneity in multifilamentary wires," IEEE Transactions on Magnetics, vol. 23, no. 2, pp. 1179-1182, 1987.

[19] R. Kimmich, A. Rimikis, and T. Schneider, "Investigation of critical current distribution in composite superconductors," IEEE Transactions on Applied Superconductivity, vol. 9, no. 2, pp. 1759-1762, 1999.

[20] A. Savitzky and M. J. Golay, "Smoothing and differentiation of data by simplified least squares procedures." Analytical Chemistry, vol. 36, no. 8 , pp. 1627-1639, 1964.

[21] H. S. Edelman and D. C. Larbalestier, "Resistive transitions and the origin of the $n$ value in superconductors with a gaussian critical-current distribution," Journal of Applied Physics, vol. 74, no. 5, pp. 3312-3315, 1993.

[22] D. Hampshire and H. Jones, "A detailed investigation of the $E-J$ characteristic and the role of defect motion within the flux-line lattice for high-current-density, high-field superconducting compounds with particular reference to data on $\mathrm{Nb}_{3} \mathrm{Sn}$ throughout its entire field-temperature phase space," Journal of Physics C: Solid State Physics, vol. 20, no. 23, p. 3533, 1987.

[23] X. Cai, A. Polyanskii, Q. Li, G. Riley, and D. Larbalestier, "Currentlimiting mechanisms in individual filaments extracted from superconducting tapes," Nature, vol. 392, no. 6679, pp. 906-909, 1998.

[24] R. Jones, E. Rhoderick, and A. Rose-Innes, "Non-linearity in the voltage-current characteristic of a type-2 superconductor," Physics Letters A, vol. 24, no. 6, pp. 318-319, 1967.

[25] M. Suenaga, A. Ghosh, Y. Xu, and D. Welch, "Irreversibility temperatures of $\mathrm{Nb}_{3} \mathrm{Sn}$ and Nb-Ti," Physical Review Letters, vol. 66, no. 13, p. 1777,1991

[26] E. H. Brandt, "The flux-line lattice in superconductors," Reports on Progress in Physics, vol. 58, no. 11, p. 1465, 1995.

[27] F. Hornung, A. Rimikis, and T. Schneider, "Current sharing and critical current distribution in Bi-2223 tapes," IEEE Transactions on Applied Superconductivity, vol. 20, no. 3, pp. 1589-1592, 2010.

[28] C. Meingast, P. Lee, and D. Larbalestier, "Quantitative description of a high $J_{\mathrm{c}} \mathrm{Nb}-\mathrm{Ti}$ superconductor during its final optimization strain. I. microstructure, $T_{\mathrm{c}}, H_{\mathrm{c} 2}$, and resistivity," Journal of Applied Physics, vol. 66, no. 12 , pp. 5962-5970, 1989. 